\title{
Configurations of Extremal Even Unimodular Lattices
}

\author{
Scott Duke Kominers
}

\begin{abstract}
We extend the results of Ozeki on the configurations of extremal even unimodular lattices. Specifically, we show that if $L$ is such a lattice of rank 56,72 , or 96 , then $L$ is generated by its minimal-norm vectors.
\end{abstract}

Mathematics Subject Classification (2000). $11 \mathrm{H} 06$ (52C07, 05B30).

Keywords. Even unimodular lattices, extremal lattices, weighted theta series.

\section{Background}

Even unimodular lattices have been studied extensively. There is a unique even unimodular lattice of rank 8, the $E_{8}$ lattice (see [2]). Niemeier [3] gave a complete characterization of the even unimodular lattices of rank 24, showing in particular that the Leech lattice $\Lambda_{24}$ is the unique extremal even unimodular lattice of rank 24. This and a similar classification problem were studied by Venkov [10, 11. Venkov [10] and Ozeki [5, 6, 7] showed a series of "configuration" results for even unimodular lattices.

Specifically, Venkov [10] showed that if $L$ is an extremal even unimodular lattice of rank 32, then $L$ is generated by its vectors of minimal norm. Ozeki [5, 7 . obtained the same result for extremal even unimodular lattices of rank 32 and showed the analogous result for extremal even unimodular lattices of rank 48 . We extend these results to cover extremal even unimodular lattices of ranks 56,72 , and 96 1

\footnotetext{
${ }^{1}$ Note that while an extremal even unimodular lattice of rank 56 has been found, the question of whether there exist even unimodular lattices of ranks 72 and 96 is still open (see [2 p. 194]).
} 


\section{Introduction}

A lattice of rank $n$ is a free $\mathbb{Z}$-module of rank $n$ equipped with a positive-definite inner product $\langle\cdot, \cdot\rangle: L \times L \rightarrow \mathbb{R}$. The dual lattice of $L$, denoted by $L^{*}$, is given by

$$
L^{*}=\{y \in L \otimes \mathbb{R} \mid\langle y, x\rangle \in \mathbb{Z} \text { for all } x \in L\} .
$$

\subsection{Even Unimodular Lattices}

A lattice $L$ is said to be integral if $\langle x, y\rangle \in \mathbb{Z}$ for all $x, y \in L$. An integral lattice $L$ is called even if all its vectors have even norm, that is, $\langle x, x\rangle \in 2 \mathbb{Z}$ for all vectors $x \in L$. An integral lattice $L$ is said to be unimodular if its dual is itself $\left(L^{*}=L\right)$.

Even unimodular lattices exist only in ranks which are multiples of eight. Sloane showed that when $L$ is even unimodular of rank $n$, the minimal norm of $L$ is bounded by

$$
\min _{\substack{x \in L \\ x \neq 0}}\{\langle x, x\rangle\} \leq 2\lfloor n / 24\rfloor+2
$$

(see [2, p. 194, Cor. 21]). A rank- $n$ even unimodular lattice is called extremal if it attains the bound (1).

\subsection{Theta Functions and Modular Forms}

Each lattice $L$ has an associated theta function $\Theta_{L}$, a generating function that encodes the norms of the vectors of $L$ in the form

$$
\Theta_{L}(z)=\sum_{x \in L} e^{\pi i z\langle x, x\rangle}
$$

where $z$ lies in the complex upper half-plane of complex numbers,

$$
\mathcal{H}=\{x \in \mathbb{C} \mid \operatorname{Im}(x)>0\} .
$$

A polynomial $P \in \mathbb{C}\left[x_{1}, \ldots, x_{n}\right]$ is called harmonic if $\Delta P=0$, where $\Delta=$ $\sum_{i=1}^{n} \frac{\partial^{2}}{\partial x_{i}^{2}}$ is the Laplace operator. For a lattice $L$ and homogeneous harmonic polynomial $P$ of degree $d$, the weighted theta series $\Theta_{L, P}$ is the function

$$
\Theta_{L, P}(z)=\sum_{x \in L} P(x) e^{\pi i z\langle x, x\rangle},
$$

where, again, $z \in \mathcal{H}$.

A holomorphic function $f: \mathcal{H} \rightarrow \mathbb{C}$ is called a modular form of weight $k$ for $S L_{2}(\mathbb{Z})$ if it is holomorphic at $i \infty$ and satisfies the condition

$$
f\left(\frac{a z+b}{c z+d}\right)=(c z+d)^{k} f(z)
$$

for all $\left(\begin{array}{ll}a & b \\ c & d\end{array}\right) \in S L_{2}(\mathbb{Z}) /\{ \pm 1\}$. If a modular form $f$ vanishes at $z=i \infty$, it is called a cusp form.

A general introduction to modular forms for $S L_{2}(\mathbb{Z})$ may be found in 9 . As shown there, if $M_{k}$ and $M_{k}^{0}$ are, respectively, the $\mathbb{C}$-vector spaces of modular forms and cusp forms of weight $k$, then $\operatorname{dim}\left(M_{k}\right)=0$ for $k$ odd, $k<0$, or $k=2$. 
Also, $\operatorname{dim}\left(M_{2 k}\right)=1$ and $\operatorname{dim}\left(M_{2 k}^{0}\right)=0$ for $4 \leq 2 k \leq 10$ and $2 k=14$. Further, multiplication by the form $\Delta=12^{-3}\left(E_{4}^{3}-E_{6}^{2}\right)$ gives an isomorphism

$$
M_{k-12} \stackrel{\sim}{\longrightarrow} M_{k}^{0}
$$

When $L$ is even unimodular of rank $n$, the theta function $\Theta_{L}$ is a modular form of weight $\frac{n}{2}$. As shown in [8], the weighted theta function $\Theta_{L, P}$ of an even unimodular lattice of rank $n$ is a cusp form of weight $\frac{n}{2}+d$, where $d>0$ is the degree of the polynomial $P$ (see [4]).

\subsection{Methods}

For an extremal even unimodular lattice $L$ having minimal norm $2 m$, the first $m-1$ coefficients of the weighted theta function $\Theta_{L, P}$ must vanish, as these coefficients count the vectors of norms less than $2 \mathrm{~m}$. We must then have

$$
\Theta_{L, P} \equiv 0
$$

for several values of $d=\operatorname{deg} P$. From this, we extract linear conditions on the vectors in $L$ which allow us to restrict the possible configurations of $L$.

\section{Dimension 72}

We adopt Ozeki's notation. For an even unimodular lattice $L$, we denote by $\Lambda_{2 m}(L)$ the set of vectors in $L$ having norm $2 m$. We denote $\left|\Lambda_{2 k}(L)\right|$ by $a(2 k, L)$. It is clear that the theta series $\Theta_{L}$ is given by $\Theta_{L}(z)=\sum_{k=0}^{\infty} a(2 k, L) q^{k}$, where $q=e^{2 \pi i z}$. We also use the notation

$$
N_{i}(x)=\operatorname{card}\left(\left\{y \in \Lambda_{m(L)}(L) \mid\langle x, y\rangle=i\right\}\right),
$$

where $m(L)$ is the minimal norm $\min \{2 k>0 \mid a(2 k, L) \neq 0\}$. Using the involution $y \longleftrightarrow-y$ of $\Lambda_{m(L)}(L)$, we see that $N_{i}(x)=N_{-i}(x)$ for any $x \in L \otimes \mathbb{R}$.

We have the following configuration result for extremal even unimodular lattices of rank 72 :

Theorem 3.1. If $L$ is an extremal even unimodular lattice of rank 72, then $L$ is generated by $\Lambda_{8}(L)$.

Proof. We partition $L$ into its equivalence classes modulo $\Lambda_{8}(L)$; to show the theorem it suffices to show that any class $[x] \in L /\left(\Lambda_{8}(L)\right)$ is represented by a vector $x_{0} \in[x]$ with $\left\langle x_{0}, x_{0}\right\rangle \leq 8$.

For any equivalence class $\left[x_{0}\right]$ where $x_{0} \neq 0$ is a representative of minimal norm, we have $\left\langle x_{0}, x_{0}\right\rangle=2 t$ for some $t \geq 5$, since we have from the fact that $L$ is extremal that $a(2, L)=a(4, L)=a(6, L)=0$. In addition, we have the inequality

$$
\left|\left\langle x_{0}, x\right\rangle\right| \leq 4
$$

for all $x \in \Lambda_{8}(L)$, because if $\left\langle x_{0}, \pm x\right\rangle>4$ then $L$ contains a vector $x \mp x_{0}$ of norm

$$
\left\langle x \mp x_{0}, x \mp x_{0}\right\rangle=\langle x, x\rangle \mp 2\left\langle x, x_{0}\right\rangle+\left\langle x_{0}, x_{0}\right\rangle<\left\langle x_{0}, x_{0}\right\rangle,
$$

contradicting the minimality of $x_{0}$ in $\left[x_{0}\right]$. 
Since $\left|\left\langle x_{0}, x\right\rangle\right| \leq 4$ for $x \in \Lambda_{8}(L)$, we have

$$
\sum_{x \in \Lambda_{8}(L)}\left\langle x, x_{0}\right\rangle^{2 k}=2 \sum_{i=1}^{4} i^{2 k} \cdot N_{i}\left(x_{0}\right),
$$

for all $k>0$.

Since $L$ is an extremal even unimodular lattice whose rank is a multiple of 24 , we know that $\Lambda_{8}(L)$ is a spherical 11-design [2, p. 196, Theorem 23]. Hence, we have that for $1 \leq k \leq 5$, the average value of the degree- $2 k$ polynomials $x \mapsto\left\langle x, x_{0}\right\rangle^{2 k}$ over $8^{-\frac{1}{2}} \Lambda_{8}(L)$ equals the average value of $x \mapsto\left\langle x, x_{0}\right\rangle^{2 k}$ over the unit sphere.

We obtain from the theta series for $L$ that $\left|\Lambda_{8}(L)\right|=6218175600$. We thus have for $1 \leq k \leq 5$

$$
\begin{aligned}
\sum_{x \in \Lambda_{8}(L)}\left\langle x, x_{0}\right\rangle^{2 k} & =\left|\Lambda_{8}(L)\right| \int_{S^{71}}\left\langle x, x_{0}\right\rangle^{2 k} d \mu(x) \\
& =6218175600 \frac{1 \cdot 3 \cdots(2 k-1)}{72 \cdot(72+2) \cdots(72+2 k-2)} 8^{k}\left\langle x_{0}, x_{0}\right\rangle^{k} \\
& =6218175600 \frac{1 \cdot 3 \cdots(2 k-1)}{72 \cdot(72+2) \cdots(72+2 k-2)} 16^{k} t^{k} .
\end{aligned}
$$

Further, the fact that $\left|\Lambda_{8}(L)\right|=6218175600$ immediately gives that

$$
6218175600=\left|\Lambda_{8}(L)\right|=N_{0}\left(x_{0}\right)+2 \sum_{i=1}^{4} N_{i}\left(x_{0}\right) .
$$

Combining this equation (41) with equations (2) and (3) for $1 \leq k \leq 5$ yields a system of six equations in the five unknowns $N_{i}\left(x_{0}\right)(0 \leq i \leq 4)$. We obtain the determinant of the (extended) $6 \times 6$ matrix for this inhomogeneous system,

$$
2^{25} 3^{9} 5^{4} 7^{2} t\left(168 t^{4}-2800 t^{3}+17745 t^{2}-50635 t+54834\right),
$$

which has no real solutions $t>0$. In particular, then, we cannot have any integral solutions for the $N_{i}\left(x_{0}\right)$ when $t>4$, so we must instead have $\left\langle x_{0}, x_{0}\right\rangle=2 t=0$, which proves the result.

\section{Dimension 96}

We continue to use the notations introduced in Section 3. We also denote by $P_{d, x_{0}}(x)$ the "zonal spherical harmonic polynomial" of degree $d$, related to the Gegenbauer polynomial by

$$
P_{d, x_{0}}(x)=G_{d}\left(\left\langle x, x_{0}\right\rangle,\left(\langle x, x\rangle\left\langle x_{0}, x_{0}\right\rangle\right)^{1 / 2}\right),
$$

where $G_{d}(t, 1)$ is the Gegenbauer polynomial of degree $d$ evaluated at $t[1$.

We find a configuration result for extremal even unimodular lattices of rank 96 analogous to our result for such lattices of rank 72 . First, however, we will need a lemma which generalizes part of Corollary 3.4 of [4]. 
Lemma 4.1. If $L$ is an extremal even unimodular lattice of rank $n$, then $\Lambda_{m}(L)$ spans $L \otimes \mathbb{R}$ for any $m>0$ such that $\Lambda_{m}(L)$ is nonempty.

Proof. We have

$$
P_{2, x_{0}}(x)=\left\langle x, x_{0}\right\rangle^{2}-\frac{\langle x, x\rangle\left\langle x_{0}, x_{0}\right\rangle}{n}
$$

spherical harmonic in dimension $n$. For fixed $x_{0} \in L \otimes \mathbb{R}$, then,

$$
\sum_{x \in \Lambda_{m}(L)} P_{2, x_{0}}(x)=0
$$

since $\Lambda_{m}(L)$ is a spherical 3-design by [12], as $L$ is an extremal even unimodular lattice of rank $n$.

Substituting the explicit form of $P_{2, x_{0}}$ into (6) gives

$$
\begin{aligned}
\sum_{x \in \Lambda_{m}(L)}\left\langle x, x_{0}\right\rangle^{2} & =\frac{1}{n}\left(\sum_{x \in \Lambda_{m}(L)}\langle x, x\rangle\right)\left\langle x_{0}, x_{0}\right\rangle \\
& =\frac{1}{n} \cdot m \cdot\left|\Lambda_{m}(L)\right|\left\langle x_{0}, x_{0}\right\rangle .
\end{aligned}
$$

If there were some $x_{0} \in L \otimes \mathbb{R}$ not in the span of $\Lambda_{m}(L)$, then the left-hand side of (17) would vanish, implying $\left|\Lambda_{m}(L)\right|=0$. By hypothesis, however, we have required $\left|\Lambda_{m}(L)\right|>0$; hence, we have the lemma.

We now proceed with the configuration theorem for rank-96 extremal even unimodular lattices:

Theorem 4.2. If $L$ is an extremal even unimodular lattice of rank 96, then $L$ is generated by $\Lambda_{10}(L)$.

Proof. We suppose that $\Lambda_{10}(L)$ does not generate $L$, and consider the set of vectors $L_{10}^{*}$ dual to the lattice $L_{10} \subsetneq L$ generated by $\Lambda_{10}(L)$. We cannot have $L_{10}^{*}=L_{10}$, since then $\Lambda_{10}(L)$ would generate an even unimodular lattice of rank 96, as its vectors span $\mathbb{R}^{96}$ by Lemma 4.1 in this case $\Lambda_{10}(L)$ would have to generate all of $L$, since $L$ is itself even unimodular of rank 96 .

Thus, there is some equivalence class $[x] \in L_{10}^{*} / L_{10}$ with minimal-norm representative $x_{0}$ having rational norm not in $2 \mathbb{Z}$. We have the inequality

$$
\left|\left\langle x_{0}, x\right\rangle\right| \leq 5
$$

for all $x \in L_{10}$, else we contradict the minimality of $x_{0}$ in $\left[x_{0}\right]$, as in the rank-72 case.

Since $x_{0} \in L_{10}^{*}$, by definition $\left\langle x, x_{0}\right\rangle \in \mathbb{Z}$ for all $x \in \Lambda_{10}(L)$. Because we also have the constraint $\left|\left\langle x_{0}, x\right\rangle\right| \leq 5$ for $x \in \Lambda_{10}(L)$, we find for all $k>0$

$$
\sum_{x \in \Lambda_{10}(L)}\left\langle x, x_{0}\right\rangle^{2 k}=2 \sum_{i=1}^{5} i^{2 k} \cdot N_{i}\left(x_{0}\right)
$$

where we recall $N_{i}\left(x_{0}\right)=\left\{x \in \Lambda_{10}(L) \mid\left\langle x, x_{0}\right\rangle=i\right\}$. 
It follows from the fact that $\Theta_{L, P_{d, x_{0}}}(z)$ is a cusp form with vanishing $q^{1}, q^{2}$, $q^{3}$, and $q^{4}$ coefficients that

$$
\Theta_{L, P_{d, x_{0}}}(z) \equiv 0
$$

for $d \in\{2,4,6,8,10,14\}$. Therefore,

$$
\sum_{x \in \Lambda_{10}(L)} P_{d, x_{0}}(x)=0
$$

for each of these $d$. (Note that for $d \in\{2,4,6,8,10\}$ we also have this result directly from the fact that $\Lambda_{10}(L)$ is a spherical 11-design, as these $P_{d, x_{0}}$ have degree at most 11.)

From the fact that $\left|\Lambda_{10}(L)\right|=565866362880$, we obtain the additional equation

$$
565866362880=\left|\Lambda_{10}(L)\right|=N_{0}\left(x_{0}\right)+2 \sum_{i=1}^{5} N_{i}\left(x_{0}\right) .
$$

Combining this equation (10) with the equations (9) for $d=\{2,4,6,8,10,14\}$, we obtain a system of seven equations in the six unknowns $N_{i}\left(x_{0}\right)(0 \leq i \leq 5)$. The determinant of this inhomogenous system's (extended) matrix factors as

$$
K s(s-12) Q(s),
$$

where $s=\left\langle x_{0}, x_{0}\right\rangle, K=-2^{42} \cdot 3^{15} \cdot 5^{10} \cdot 7^{5} \cdot 11 \cdot 13 \cdot 17 \cdot 19 \cdot 29 \cdot 47 \cdot 53 \cdot 59$, and $Q(s)=25 s^{5}-1275 s^{4}+26112 s^{3}-267444 s^{2}+1362720 s-2741760$ is an irreducible quintic polynomial. The only rational $s=\left\langle x_{0}, x_{0}\right\rangle$ which give solutions to our system, therefore, are the roots

$$
s=\left\langle x_{0}, x_{0}\right\rangle \in\{0,12\}
$$

of the determinant (11).

But then, any $x \in\left[x_{0}\right]$ is of the form $x_{0}+x_{1}$ for some $x_{1} \in L_{10}$, so we see that

$$
\langle x, x\rangle=\left\langle x_{0}, x_{0}\right\rangle+2\left\langle x_{0}, x_{1}\right\rangle+\left\langle x_{1}, x_{1}\right\rangle .
$$

Since $\left\langle x_{0}, x_{0}\right\rangle \in\{0,12\} \subset 2 \mathbb{Z},\left\langle x_{0}, x_{1}\right\rangle \in \mathbb{Z}$, and $\left\langle x_{1}, x_{1}\right\rangle \in 2 \mathbb{Z}$, we see that $\langle x, x\rangle \in$ $2 \mathbb{Z}$, from which it follows that $L_{10}^{*}$ is even.

In particular, then, $L_{10}^{*}$ is integral, whence it has integral discriminant. Because $L_{10}$ has finite index $\left[L_{10}: L\right]$ in $L$, we see that $L_{10}$ must have positive integer discriminant

$$
\operatorname{disc}\left(L_{10}\right)=\left[L_{10}: L\right]^{2} \operatorname{disc}(L)=\left[L_{10}: L\right]^{2},
$$

as well. But then, we have both

$$
\begin{aligned}
{\left[L_{10}: L\right]^{2} } & \in \mathbb{Z} \\
{\left[L_{10}: L\right]^{-2} } & \in \mathbb{Z},
\end{aligned}
$$

from which it follows that $\left[L_{10}: L\right]=1$, so that $L_{10}=L$. 


\section{Dimension 56}

We use a combination of the techniques developed in Sections 3 and 4 to find a configuration result for extremal even unimodular lattices of rank 56.

Theorem 5.1. If $L$ is an extremal even unimodular lattice of rank 56, then $L$ is generated by $\Lambda_{6}(L)$.

Proof. We partition $L$ into equivalence classes modulo $\Lambda_{6}(L)$. As in the rank-72 case, it suffices to show that any class $[x] \in L /\left(\Lambda_{6}(L)\right)$ is represented by a vector $x_{0} \in[x]$ with $\left\langle x_{0}, x_{0}\right\rangle \leq 6$.

Let $x_{0}$ a minimal representative of any equivalence class $\left[x_{0}\right] \in L /\left(\Lambda_{6}(L)\right)$. We have $\left\langle x_{0}, x_{0}\right\rangle=2 t$ for some $t \geq 4$ if $x_{0} \neq 0$ and we have the inequality

$$
\left|\left\langle x_{0}, x\right\rangle\right| \leq 3
$$

for all $x \in \Lambda_{6}(L)$.

From this condition, we obtain the equation

$$
\sum_{x \in \Lambda_{6}(L)}\left\langle x, x_{0}\right\rangle^{2 k}=2 \sum_{i=1}^{3} \cdot i^{2 k} \cdot N_{i}\left(x_{0}\right),
$$

for all $k>0$.

It follows from the fact that $\Theta_{L, P_{d, x_{0}}}(z)$ is a cusp form with vanishing $q^{1}$ and $q^{2}$ coefficients that

$$
\Theta_{L, P_{d, x_{0}}}(z) \equiv 0
$$

for $d \in\{2,4,6,10\}$. This gives

$$
\sum_{x \in \Lambda_{6}(L)} P_{d, x_{0}}(x)=0
$$

for each $d \in\{2,4,6,10\}$.

Combining the fact that

$$
N_{0}\left(x_{0}\right)+2 \sum_{i=1}^{3} N_{i}\left(x_{0}\right)=\left|\Lambda_{6}(L)\right|=15590400
$$

with (13) and (14) for $d \in\{2,4,6,10\}$, we obtain a system of five equations in the four unknowns $N_{i}\left(x_{0}\right)(0 \leq i \leq 3)$. This system's (extended) matrix has determinant

$$
-K t^{2} Q(t)
$$

where $K=2^{28} \cdot 3^{8} \cdot 5^{3} \cdot 7 \cdot 29 \cdot 31$ and $Q(t)$ is the irreducible sextic

$$
Q(t)=8976 t^{6}-76120 t^{5}+104624 t^{4}+533337 t^{3}-972400 t^{2}-1952280 t+3644256 .
$$

Thus, the only integral solution to the system is $t=0$. But then we cannot have $\left\langle x_{0}, x_{0}\right\rangle=2 t>6$, so we are done. 


\section{Acknowledgements.}

This work was partially supported by a fellowship from the 2006 Harvard College Program for Research in Science and Engineering.

The author is extremely grateful to Professor Noam D. Elkies, under whose direction this research was conducted, for his instruction, commentary on the work, and remarks on several earlier drafts of this article. He also thanks Gabriele Nebe and an anonymous referee for their helpful comments and suggestions on earlier drafts of this article.

\section{References}

[1] C. Bachoc, G. Nebe, B. Venkov, Odd unimodular lattices of minimum 4, Acta Aritmetica 101(2) (2002).

[2] J. H. Conway, N. J. A. Sloane, Sphere Packing, Lattices and Groups, 3rd edition (Springer-Verlag, 1999).

[3] H.-V. Niemeier, Definite quadratische Formen der Dimension 24 und Diskriminante 1 (German), J. Number Thy. 5 (1973) 142-178.

[4] W. Ebeling, Lattices and Codes, 2nd edition (Vieweg, 2002).

[5] M. Ozeki, On even unimodular positive definite quadratic lattices of rank 32, Math. Z. 191(2) (1986) 283-291.

[6] M. Ozeki, On the structure of even unimodular extremal lattices of rank 40, Rocky Mtn. J. Math. 19(3) (1989) 847-862.

[7] M. Ozeki, On the configurations of even unimodular lattices of rank 48, Arch. Math. 46(1) (1986) 247-287.

[8] A. Schoeneberg, Das verhalten von mehrfachen thetareihen bei modulsubstitutionen (German), Math. Ann. 116(1) (1939) 511-523.

[9] J.-P. Serre, A Course in Arithmetic (Springer-Verlag, 1973).

[10] B. B. Venkov, Even unimodular Euclidean lattices of dimension 32 (Russian), Zap. Nauchn. Sem. Leningrad. Otdel. Math. Inst. Steklov (LOMI) 116 (1982) 44-55.

[11] B. B. Venkov, On the classification of integral even unimodular 24-dimensional quadratic forms, Proc. Steklov Inst. Math. 148 (1980) 63-74.

[12] B. B. Venkov, Réseaux et designs sphériques (French), in Réseaux Euclidiens, Designs Sphériques et Formes Modulaires, Monogr. Enseign. Math., Vol. 37 (Enseignement Math., 2001), pp. 10-86.

Scott Duke Kominers

Department of Mathematics, Harvard University

c/o 8520 Burning Tree Road, Bethesda, MD, 20817

e-mail: kominers@fas .harvard.edu 